\title{
Interband Cascade Lasers for the Mid-Infrared Spectral Region
}

\author{
S. Höfling*, R. Weih, M. Dallner, and M. Kamp \\ Technische Physik, Physikalisches Institut, Universität Würzburg and Wilhelm Conrad Röntgen \\ Research Center for Complex Material Systems, Universität Würzburg, Am Hubland, D-97074 \\ Würzburg, Germany
}

* present address: SUPA, School of Physics and Astronomy, University of St Andrews, St Andrews, KY16 9SS, United Kingdom

e-mail: sven.hoefling@physik.uni-wuerzburg.de

\begin{abstract}
Interband cascade lasers are mid-infrared semiconductor lasers that are very promising for low power consumption applications in the 3-6 $\mu \mathrm{m}$ spectral range. Therefore, they are ideal for gas sensing of hydrocarbons. Combining interband transitions utilized in diode lasers with a cascading scheme widely exploited in intersubband transition based quantum cascade lasers, interband cacscade lasers can operate at threshold current densities around or below $100 \mathrm{Acm}^{-2}$ at room temperature. Distributed feedback interband cascade lasers emit at room temperature continuous wave output powers in the $\mathrm{mW}$ range and above, and their side mode suppression ratio can well exceed $20 \mathrm{~dB}$.
\end{abstract}

Keywords: semiconductor laser, interband cascade laser, mid infrared

\section{INTRODUCTION}

The mid-infrared spectral region is ideal for gas sensing because various industrially and environmentally important gases have their strongest absorption features in this spectral range. Semiconductor lasers are compact, reliable and can emit in single mode at room temperature which makes them very suitable light sources for highly sensitive tunable laser spectroscopy. Different semiconductor laser concepts are utilized to cover the mid-infrared spectral range with single mode semiconductor lasers, namely diode lasers, quantum cascade lasers and interband cascade lasers [1]. Conventional semiconductor diode lasers on GaSb substrate can cover wavelengths up to about $3.7 \mu \mathrm{m}[2,3]$. Quantum cascade lasers can cover a very wide range of the mid-infrared and deliver high output powers and wide wavelength tunability [4,5]. They rely on intersubband transitions of electrons in a stair case of confined energy levels formed in the conduction band of multi-quantum well structures. The very short non-radiative lifetime dominated by longitudinaloptical phonon scattering results however in room temperature threshold current densities on the order of $\mathrm{kAcm}^{-2}$ or more. Interband cascade lasers (ICLs) [6] in contrast can exhibit very low threshold current densities down to $100 \mathrm{Acm}^{-}$ ${ }^{2}[7,8]$ and cover the wavelength range between about $3.0-5.5 \mu \mathrm{m}$ continuous wave room temperature eoperating lasers $[9,10]$. ICLs exploit like quantum cascade lasers stair case like radiative transition, but between electrons and holes in special type II band alignment. In the following manuscript, we describe design principles and recent progress made in our group on ICL research.

\section{INTERBAND CASCADE LASER DESIGN}

The ICLs presented in this manuscript have been grown by molecular beam epitaxy on Tellurium doped (100) GaSb substrates. Figure 1 shows schematically shows a typical ICL layer structure. The orange coloured part in the centre of the figure the represents the cascaded active region. Since the refractive index of the active region is not notably higher

Novel In-Plane Semiconductor Lasers XIII, edited by Alexey A. Belyanin,

Peter M. Smowton, Proc. of SPIE Vol. 9002, 90021B - (c) 2014 SPIE

CCC code: $0277-786 X / 14 / \$ 18 \cdot$ doi: $10.1117 / 12.2038130$

Proc. of SPIE Vol. 9002 90021B-1 
than the refractive index of the AlSb/InAs:Si superlattice cladding layers, the active region I sandwiched between $\mathrm{GaSb}$ separate confinement layers (SCLs). This increases the overlap of the optical waveguide mode overlap with the active region and therwith enhances the gain of the laser. The functional parts of the laser structure are connected with graded AlSb/InAs:Si SL-transition layers to effectively smoothen conduction band offsets and therewith reduce operation voltages. The epitaxial growth is finalized with a highly doped InAs:Si layer to enable the fabrication oflow resitance contacts by metallization. The left part of figure 1 shows a transmission electron microscopy images of an active region design section. As it can bee seen from figure 1 at the right hand side, sharp heterostructure interfaces are obtained.

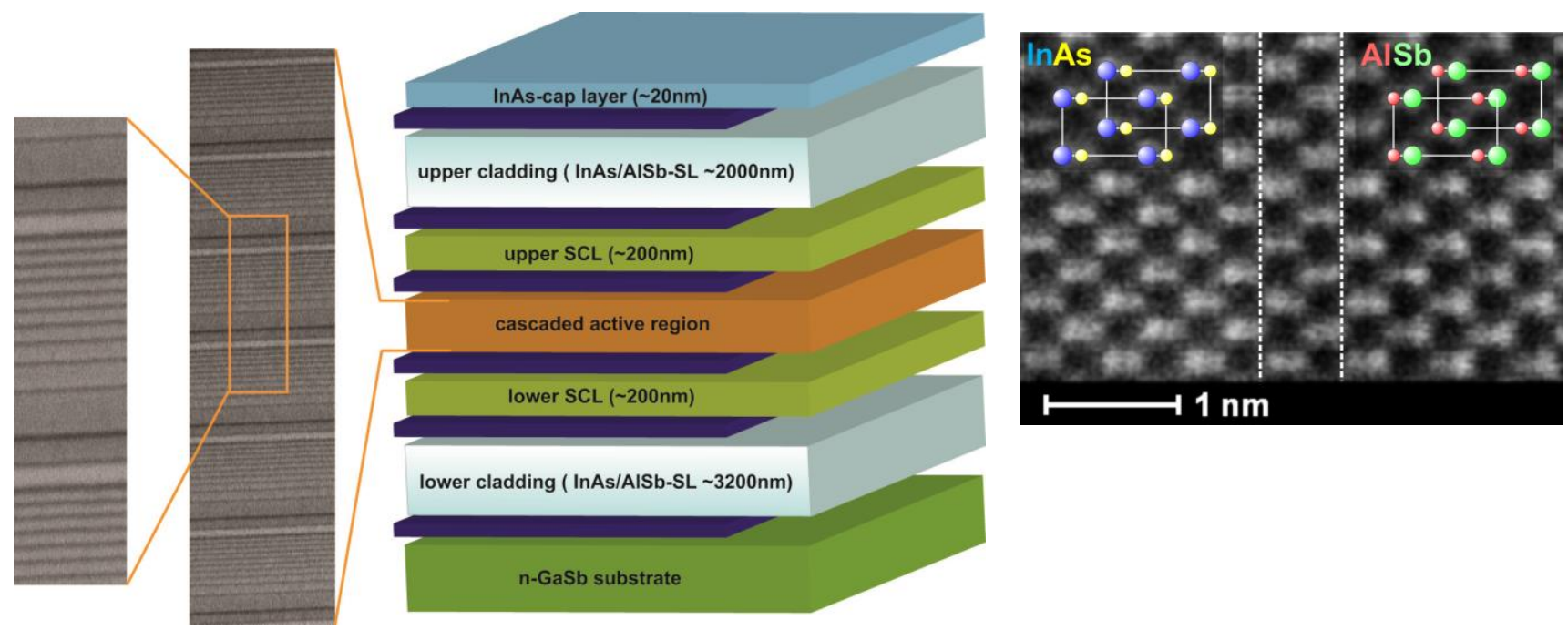

Figure 1 Left: Transmission electron microscope image of an ICL cascaded active region. Centre: schematics of an typical interband cascade laser epitaxiual layer design on GaSb substrarte. Right: Scanning transmission electron microscope image of an InAs/AlSb interface together with a cartoon of the InAs and AlSb lattice along the (110) axis. Material intermixing at the interface is minimized to about one lattice constant.

The unique band structure of the GaInSb InAs enables the cascading of interband tranistions in ICL structures. The conduction band of InAs resides about $200 \mathrm{meV}$ lower in energy than the valence band of GaSb. In figure 2 a cascade of an ICL active region is shown under an electric field of $92 \mathrm{kV} / \mathrm{cm}$. The black and red lines represent the band structure while the blue and orange lines represent the probability densities of most relevant electron and hole states. At the hole injector-electron injector interface, a so called semimetallic interface or leaky quantum well result sin the generation of electrons and hols by tunnelling. This type of internal carrier generation is a key feature of QCLs. All layers of the displayed structure add up to a cascade length of $42 \mathrm{~nm}$. One stage of an active region can be divided into three main parts, the electron injector, the hole injector and the active $\mathrm{W}$-shaped quantum well (W-QW).

The radiative transition takes place between electrons confined in InAs quantum wells and holes confined in GaInSb. The so called W-quantum well (W_QW) design enhances the spatial overlap between electron and hole wavefunctions and therwith increases the laser gain. 


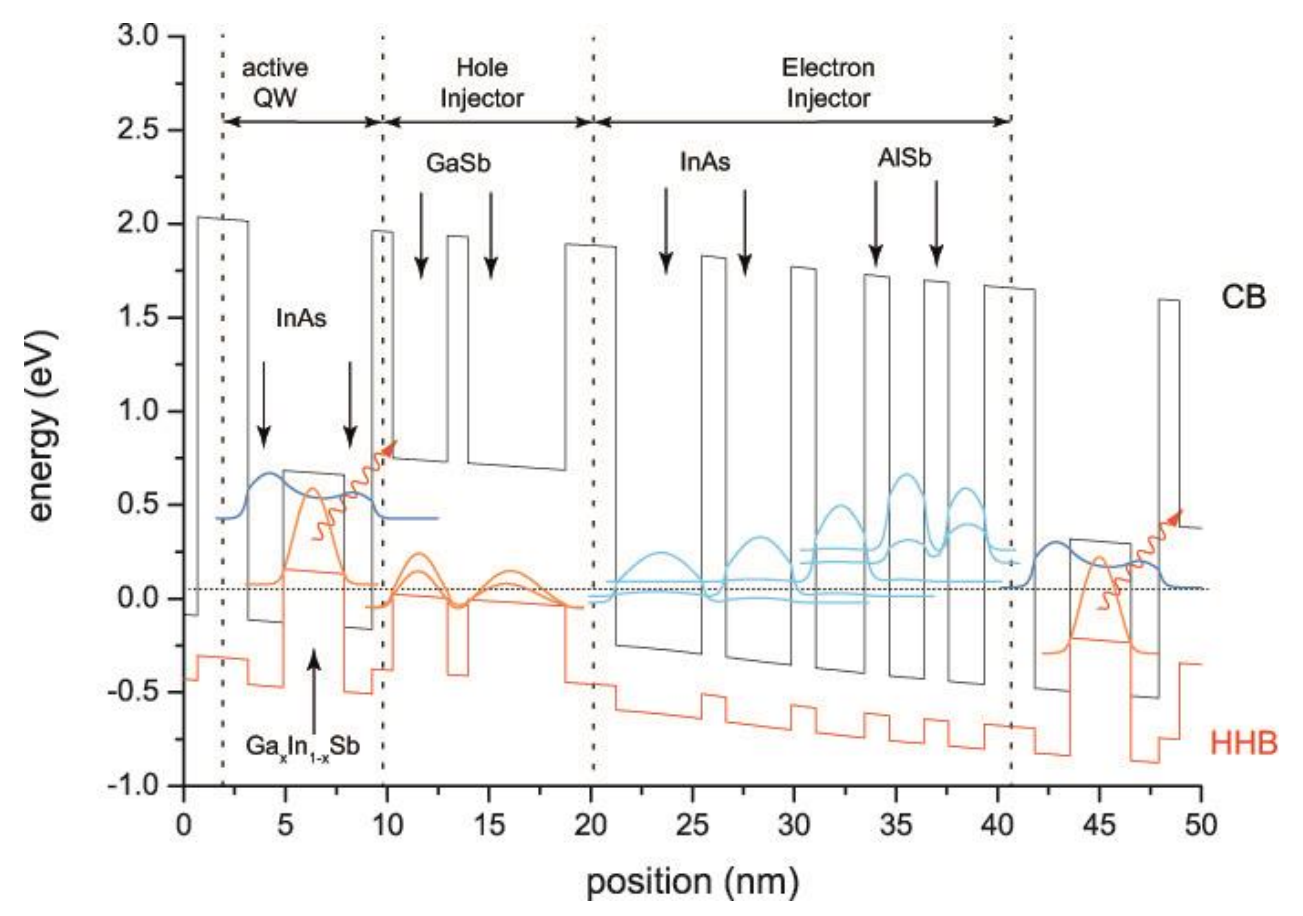

Figure 2 Band structure profile and absolute squared wavefunctions of most relevant electron and hole states in one stage of an ICL active region under an applied electric field of $92 \mathrm{kV} / \mathrm{cm}$. The different functional parts labeled and are separated by vertical dashed lines. CB refers to conduction band and HHB to heavy hole band.

\section{LASER CHARACTERISTICS}

Based on the epitaxial layers, broad area lasers were processed. $150 \mu \mathrm{m}$ wide ridges were lithographically defined and dry etched through the active region into the lower cladding. Then, $200 \mathrm{~nm}$ of each $\mathrm{Si}_{3} \mathrm{~N}_{4}$ and $\mathrm{SiO}_{2}$ were sputtered to passivate the side walls. Afterwards a contact window was opened on top of the ridge and a Ti/Pt/Au contact was evaporated. The substrate was thinned to approximately $150 \mu \mathrm{m}$ and a $\mathrm{Au} / \mathrm{Ni} / \mathrm{AuGe}$-contact was evaporated on the backside. An ICL with 10 active stages emitting around $3.6 \mu \mathrm{m}$ was grown and characterized as presented in figure 3 . The threshold current of this device is less than 0.3 A corresponding to a record threshold current density of only 98 $\mathrm{A} / \mathrm{cm}^{2}$.

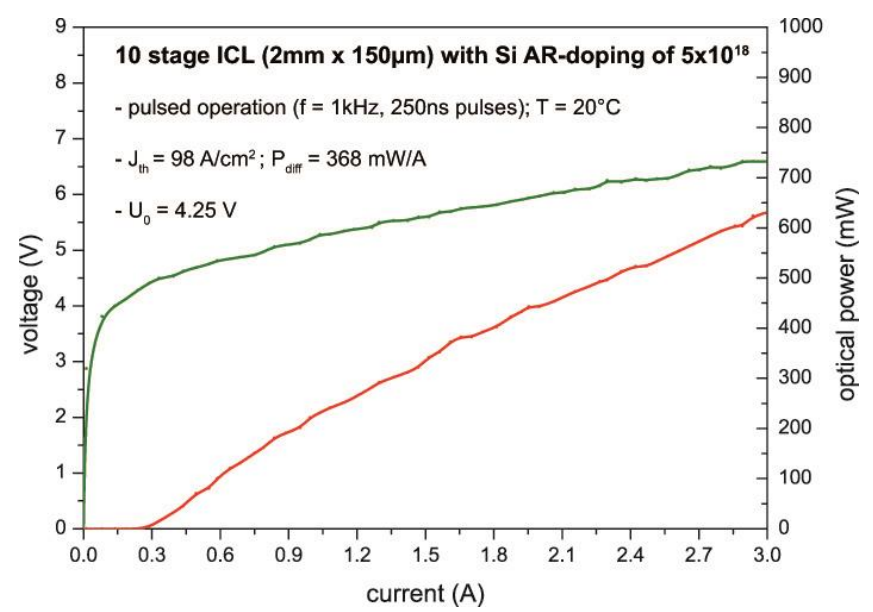

Figure 3 Light-current and voltage-current characteristic of a 10 stage broad area $(2 \mathrm{~mm}$ x $150 \mu \mathrm{m})$ ICL that was etched though the active region. The threshold current density is as low as $98 \mathrm{~A} / \mathrm{cm}^{2}$ at room temperature. AR: active regsion. 


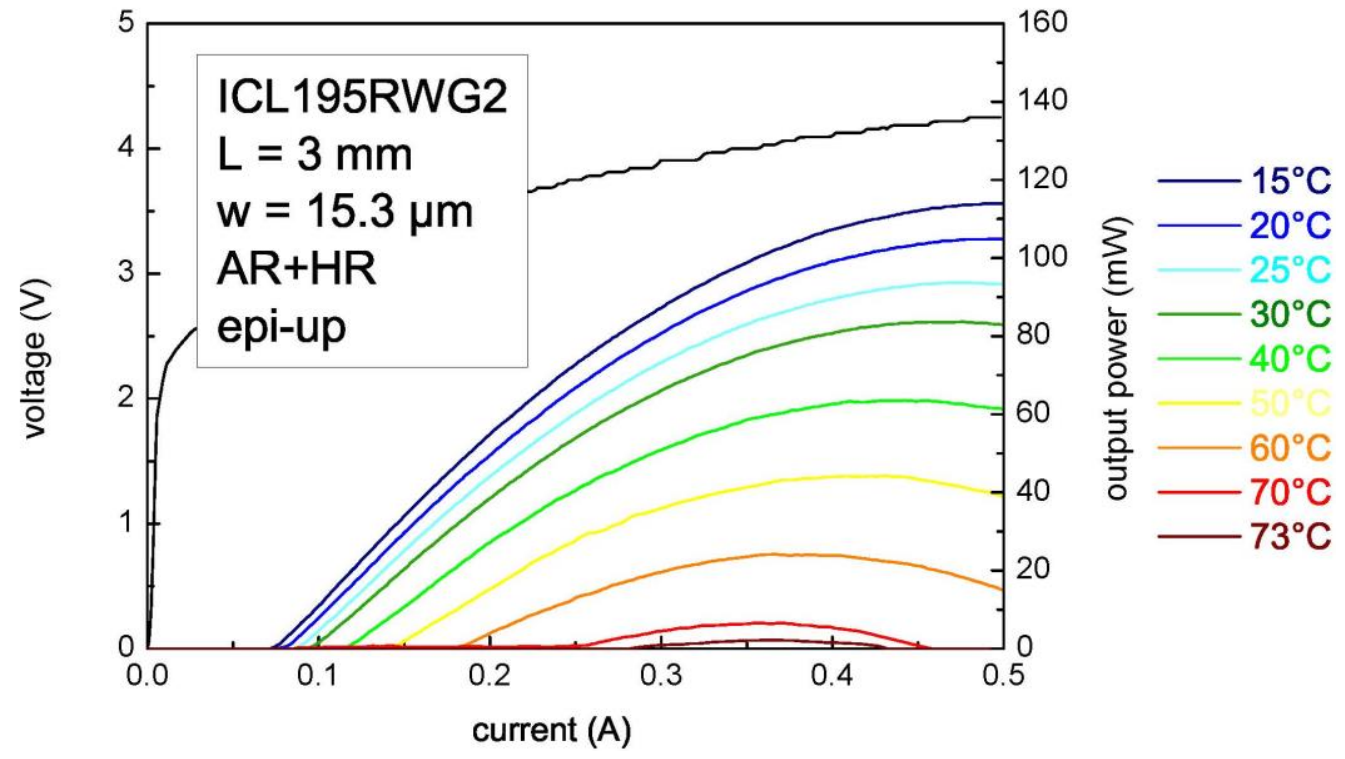

Figure 4 Temperature dependent continuous wave light-output characteristic of a ridge waveguide ICL with $15.3 \mu \mathrm{m}$ ridge width and $3 \mathrm{~mm}$ length. AR: anti-reflection coating. HR: high-reflection coating.

To evaluate the potential of the fabricated epitaxial layers for continuous wave mode narrow ridge waveguide lasers were processed. The process was basically the same as for the broad area lasers. For electro-optical characterization The front facet has been anti-reflection coated and the rear facet coated with a high reflection metal coating consisting of $\mathrm{Al}_{2} \mathrm{O}_{3}$ as insulator and gold. Afterwards the laser was mounted on a copper heat sink. Temperature dependent continuous wave light-output characteristic of a ridge waveguide ICL with $15.3 \mu \mathrm{m}$ ridge width and $3 \mathrm{~mm}$ length are presented in figure 4 . The ICL operates in continuous wave mode up to $73{ }^{\circ} \mathrm{C}$ and the front facet output power at room temperature for the epi-side up mounted device exceeds $100 \mathrm{~mW}$.

Gas sensing application of ICLs require emission at a single mode to scan over absorption lines. For this reason ridge waveguide lasers with fourth order vertical sidewall gratings were processed applying inductive coupled plasma dry etching. The grating provides wavelength selective feedback and suppresses higher order lateral modes [11]. In figure 5 a single mode spectrum of a laser with vertical sidewall grading is shown. The side mode suppression ratio exceeds $30 \mathrm{~dB}$ while the temperature tuning rate was extracted to $0.5 \mathrm{~nm} / \mathrm{K}$ and the current tuning rate was $0.15 \mathrm{~nm} / \mathrm{K}$. This device is therewith ideally suitable for gas detection of NO. 


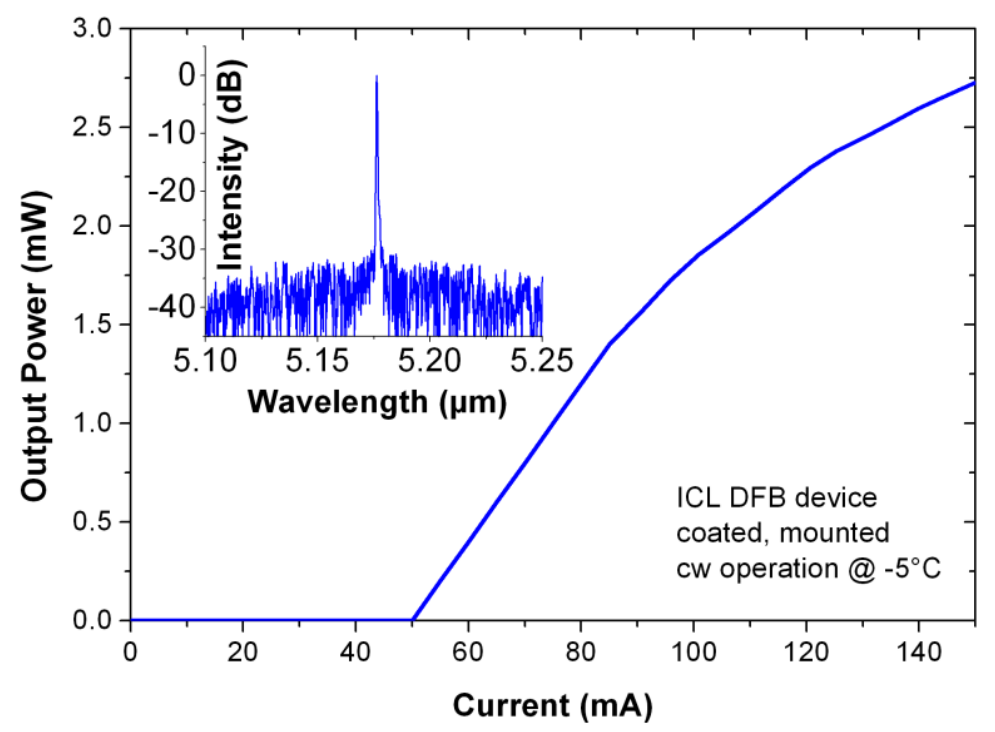

Figure 5 Light-current characteristics and spectrum (inset) of a single mode emitting distributed feedback ICLs emitting around $5.2 \mu \mathrm{m}[12]$.

The electro-optical characteristic of an ICL with certical side wall gratings to provide distributed feedback and therwith select a single emission mode is represented in figure 5 . At a temperature of $-5^{\circ} \mathrm{C}$, continuous wave laser operation is observed on a thermo-electrically cooled TO header. The inset shows the spectral characteristic of the laser emiting at around $5.2 \mu \mathrm{m}$ with a signal to noise ratios around $30 \mathrm{~dB}$. The device emits ample power for tunable laser absorption spectroscopy [13] with a threshold power consumption of $138 \mathrm{~mW}$.

\section{CONCLUSION}

Interband cascade laser data has been presented that show the suitability of these lasers for highly sensitive gas detection. Single mode operation of distributed feedback ICLs with side mode suppression of about $30 \mathrm{~dB}$ was observed. ICLs seem to be the best choice to cover the 3-5 $\mu \mathrm{m}$ wavelength range with semiconductor lasers for gas sensing if low power consumption for operation is required.

\section{ACKNOWLEDGEMENTS}

We thank A. Wolf, M. Wagenbrenner, S. Handel, S. Kuhn and T. Steinl for technical assistance and M. von Edlinger, J. Scheuermann, C. Zimmermann, L. Nähle, M. Fischer and J. Koeth for the fruitful collaboration on distributed feedback laser realization. Financial support of this work by the German Ministry of Education and Research (FKZ: 13N12440) and by the European Commission in the framework of the FP7 project 'WideLase' (grant no. 318798 ) is gratefully acknowledged. 


\section{REFERENCES}

[1] A Bauer, K Rößner, T Lehnhardt, M Kamp, S Höfling, L. Worschech and A Forchel; "Mid-infrared semiconductor heterostructure lasers for gas sensing applications", Semicond. Sci. Technol. 26 , 014032 (2011).

[2] K.Vizbaras and M.-C.Amann; "Room-temperature $3.73 \mu \mathrm{m}$ GaSb-based type-I quantum well lasers with quinternary barrierss" Semicond. Sci. Technol. 27 (2012).

[3] T.Hosoda, G.Kipshidze, L.Shterengas and G.Belenky; "Diode lasers emitting near $3.44 \mathrm{~mm}$ in continuous-wave regime at 300K", Electronics Letters 46, 21 (2008).

[4] Yu Yao, Anthony J. Hoffman and Claire F. Gmachl; "Mid-infrared quantum cascade lasers", Nature Photonics 6, 433 (2012).

[5] N.Bandyopadhyay, S.Slivken, Y.Bai, and M.Razeghi; High power, continuous wave, room temperature operation of $\lambda \sim 3.4 \mu \mathrm{m}$ and $\lambda \sim 3.55 \mu \mathrm{m}$ InP-based quantum cascade lasers", Applied Physics Letters 100, 212104 (2012)

[6] R.Q.Yang;"'Infrared laser based on intersubband transitions in quantum wells" Superlattices and Microstructures, Vol.17, No.1 (1995)

[6] R.Q.Yang;,"Infrared laser based on intersubband transitions in quantum wells" Superlattices and Microstructures, Vol.17, No.1 (1995)

[7] R. Weih, M. Kamp and S. Höfling; "Interband cascade lasers with room temperature threshold currentdensities below 100 A/cm2”, Appl. Phys. Lett. 102, 231123 (2013)

[8] I.Vurgaftman, W.W.Bewley, C.L.Canedy, C.S.Kim, M.Kim, C.D.Merritt, J.Abell, J.R.Lindle and J.R.Meyer; "Rebalancing of internally generated carriers for mid-infrared interband cascade lasers with very low power consumption", Nature Communications, DOI: 10.1038/ncomms1595 (2011).

[9] W.W.Bewley, C.L.Canedy, C.S.Kim, M.Kim, C.D.Merritt, J.Abell, I.Vurgaftman, and J.R.Meyer, "Continuouswave interband cascade lasers operating above room temperature at $\lambda=4.7-5.6 \mu \mathrm{m}$ ", Optics Express, Vol.20 No.3 (2012).

[10] I. Vurgaftman, W. W. Bewley, C. L. Canedy, C. S. Kim, M. Kim, C. D. Merritt, J. Abell, and Jerry R. Meyer,, "Interband Cascade Lasers With Low Threshold Powers and High Output Powers", IEEE J. of Sel. Top. In Quant. Electr. 19, 1200210 (2013).

[11] C.S.Kim, M.Kim, W.W.Bewley, J.R.Lindle, C.L.Canedy, J.Abell, I.Vurgaftman and J.R.Meyer; "Corrugatedsidewall interband cascade lasers with single-mode midwave-infrared emission at room temperature ", Applied Physics Letters 95, 231103 (2009).

[12] M. von Edlinger, J. Scheuermann, R. Weih, C. Zimmermann, L. Nähle, M. Fischer, J. Koeth, S. Höfling, and M. Kamp; "Monomode Interband Cascade Lasers at 5.2 um for Nitric Oxide Sensing”, IEEE Phot. Technol. Lett., DOI 10.1109/LPT.2013.2297447 (20013)..

[13] S.Lundqvist, P.Kluczynski, R.Weih, M.v.Edlinger, L.Nähle, M.Fischer, A.Bauer, S.Höfling, J.Koeth; "Sensing of formaldehyde using a distributed feedback interband cascade laser emitting around 3493nm", Applied Optics, 51, 6009 (2012). 